\title{
Interroger les fables et les contes pour développer le jugement moral
}

Questioning Fables and Stories to Develop Moral Judgment

\section{Claudine Leleux}

\section{OpenEdition}

Journals

Édition électronique

URL : http://journals.openedition.org/recherchestravaux/1640

DOI : 10.4000/recherchestravaux.1640

ISSN : 1969-6434

Éditeur

UGA Éditions/Université Grenoble Alpes

Édition imprimée

ISBN : 978-2-37747-098-3

ISSN : 0151-1874

Référence électronique

Claudine Leleux, «Interroger les fables et les contes pour développer le jugement moral », Recherches \& Travaux [En ligne], 94 | 2019, mis en ligne le 20 juin 2019, consulté le 08 septembre 2020. URL: http://journals.openedition.org/recherchestravaux/1640; DOI : https://doi.org/10.4000/ recherchestravaux.1640

Ce document a été généré automatiquement le 8 septembre 2020

(C) Recherches \& Travaux 


\title{
Interroger les fables et les contes pour développer le jugement moral
}

Questioning Fables and Stories to Develop Moral Judgment

\author{
Claudine Leleux
}

1 Cette contribution vise à montrer que les fables et les contes, parce qu'ils appartiennent à un genre littéraire particulier, sont des supports idéaux à partir desquels l'enseignant peut développer le jugement moral des élèves et ainsi rencontrer l'une des finalités de l'enseignement moral et civique (EMC).

2 Les fables et les contes constituent en effet des récits, au départ oraux, pour transmettre à autrui la «morale» d'une histoire. Ce sont des récits édifiants qui condensent les leçons de l'expérience et fournissent à l'auditeur puis au lecteur un savoir pratique.

3 L'enseignement moral et civique, comme tout enseignement, vise la transmission d'un savoir mais la spécificité de l'EMC, par rapport aux autres disciplines, vise la transmission d'un savoir pratique formé de jugements normatifs (portant sur les normes d'action) et évaluatifs (portant sur les valeurs, les goûts et les préférences).

4 Je me propose dans cet article successivement de :

1. distinguer valeurs et normes souvent confondues dans le langage quotidien et différencier ainsi jugement normatif et jugement évaluatif;

2. montrer la spécificité de l'enseignement moral et civique ;

3. souligner que les questions pratiques peuvent prétendre à une vérité, à condition de préciser ce que l'on entend par ce terme de " vérité »;

4. rappeler les deux grandes théories de développement du jugement normatif et l'état de mes recherches en ce domaine ;

5. souligner le rôle de la littérature et, en particulier, des fables et des contes, pour développer le savoir pratique. 


\section{Distinguer valeurs et normes}

5 Dans le langage courant, l'habitude nous pousse généralement à confondre valeur et norme. La norme, du latin norma ${ }^{1}$, est l'énoncé d'une règle d'action, véhiculant, de manière implicite ou explicite, une obligation en vue d'atteindre un résultat: on parlera ici de «norme technique » lorsque le résultat vise un but pratique (efficacité de l'action), de «norme éthique " lorsque le résultat vise une finalité éthique (réussir sa vie) ou de «norme morale » quand l'obligation se présente à tous sous la forme d'un impératif catégorique visant à préserver l'humanité de l'homme ${ }^{2}$. Toutes ces normes, parce qu'elles sont relatives aux mœurs (en latin mores), peuvent être appelées «normes de moralité ». Lorsque ces normes sont coulées dans la forme du droit (juris), nous les nommerons « normes juridiques».

6 En revanche, la valeur se présente comme un concept qui condense ou synthétise une norme. Ainsi, «Liberté » condense par exemple la norme juridique "J'ai droit à la liberté ».

7 Si la valeur est un concept, tout concept n'est pas une valeur. La valeur est un concept particulier qui se présente comme un indicateur de sens.

- Sens au sens de signification. La valeur de la vie, par exemple, peut signifier, pour le croyant d'une religion du Livre, la vie biologique alors que pour certains athées ou agnostiques, c'est la vie humaine qui est la valeur.

- Sens au sens de direction. La valeur oriente nos choix et nos comportements.

- Sens au sens de finalité, de sens de notre existence. Ainsi certaines valeurs seront plus dignes d'être poursuivies selon que l'on veuille fonder une famille, s'enrichir ou gagner son paradis.

- Enfin, sens au sens de sensible parce que nous sommes attachés aux valeurs alors que nous ne le sommes pas aux autres concepts comme « insecte » ou « meuble ». Cet attachement est antérieur au langage, il est le fruit d'une expérience sensible satisfaisante qui demande à être reproduite. Dès son plus jeune âge, en effet, l'enfant apprend à discriminer ce qui est digne d'être vécu et recherché et, au contraire, ce qu'il lui faut éviter parce que source de peine, de souffrance ou de désagrément.

8 Si la valeur est un indicateur de sens, cela signifie qu'elle ne peut faire l'objet que d'une préférence individuelle, qu'il n'y a, autrement dit, de hiérarchie axiologique que subjective. Cela signifie donc qu'il est impensable de vouloir transmettre des valeurs, fussent-elles de la République. Tout au plus, pouvons-nous transmettre des normes qui engagent tous les citoyens ${ }^{3}$.

9 J'insiste sur ce point : il est pédagogiquement utile d'amener les élèves à clarifier leurs propres valeurs et même à les hiérarchiser dans un contexte donné pour leur apprendre à mieux se connaître et à faire l'expérience du pluralisme des valeurs. En revanche, il n'est pas possible de les faire adhérer à des valeurs qui font l'objet d'une élection subjective et qui dépendent du sens qu'ils donnent à leur existence.

Travailler les valeurs en classe vise donc à travailler le fameux proverbe «Connais-toi toi-même » du fronton du temple de Delphes et à développer le jugement évaluatif qui doit permettre à l'élève d'exprimer sa préférence d'une valeur sur une autre et de découvrir que les autres n'ont pas forcément la même échelle de valeurs que la sienne. Ce travail sur les valeurs peut se faire pour les plus petits à partir d'images ${ }^{4}$ ou à partir d'albums ${ }^{5}$, de contes ou de fables. Quand les élèves sont au Collège et au Lycée, 
l'enseignant peut les confronter à des textes d'auteur par lesquels ils feront l'expérience de la différence entre la valeur préconisée par un auteur et les leurs ${ }^{6}$. Ainsi en classe de seconde, les élèves découvrent, par exemple, que Nietzsche ne défend pas nécessairement les mêmes valeurs qu'eux, lorsqu'il pourfend l'égalité et défend un point de vue aristocratique ${ }^{7}$.

\section{Enseignement moral et civique}

11 Ce qui est transmissible et peut faire l'objet d'un enseignement, comme le prévoit l'intitulé du cours Enseignement moral et civique (EMC), c'est un savoir. Mais de quel savoir parlons-nous dans ce cas?

Pour faire simple, nous devons discerner deux types de savoirs au moins : le savoir théorique et le savoir pratique, la distinction qu'opérait déjà Aristote dans l'antiquité

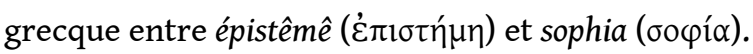

Ainsi les quatre énoncés suivants font-ils l'objet d'un savoir théorique; ce sont des énoncés descriptifs ou constatifs :

- Les insectes ont 3 paires de pattes; les arachnides, 4 ;

$\cdot 2+2=4$;

- Ce fleuve se divise en deux ;

- La morale d'Aristote est eudémoniste...

Tandis que :

- Il faut dire la vérité pour garder la confiance (valeurs de vérité et de confiance);

- Il faut considérer que les hommes sont égaux quels que soit leur sexe, leur religion... (valeur d'égalité);

- Il est interdit de voler (valeur de propriété).

font l'objet d'un savoir pratique ; ce sont des énoncés normatifs ou prescriptifs.

Lorsqu'on se meut dans l'exercice et le développement du jugement normatif, nous quittons le terrain scientifique des disciplines, des énoncés descriptifs et constatifs sur lesquels la communauté des savants s'est mise d'accord, du moins provisoirement, pour entrer dans le champ des énoncés normatifs et prescriptifs qui constituent le corpus des disciplines de la morale/l'éthique et du droit. Énoncés dont la validité doit être interrogée. Nous allons y revenir.

Pour le dire encore autrement, l'enseignement de la plupart des disciplines consiste à transmettre un savoir sur ce qui est alors que l'EMC consiste à transmettre un savoir sur ce qui doit être (un jugement normatif) ou sur ce qui vaut et est souhaitable (un jugement évaluatif) ${ }^{8}$. Notons que "juger» veut souvent dire évaluer, coter (approuver ou blâmer), d'où son caractère parfois péjoratif. Le concept de "jugement » est pris ici dans son sens philosophique, celui d'affirmer ou nier un rapport entre un sujet et un attribut. Un jugement normatif est un jugement qui énonce une norme. En fonction d'une finalité donnée (but, télos ou fin de l'espèce/règne des fins), en se fondant sur l'expérience, il est possible d'énoncer, de dégager, des leçons de savoir-vivre par subsomption.

Exemple :

Si mentir peut entraîner la perte de confiance (raison de ne pas mentir);

Si Socrate ment ;

Alors Socrate risque qu'on ne lui fasse plus confiance (devoir de ne pas mentir). 

goûts ou deux valeurs. Ainsi, par exemple, l'énoncé qu'on a attribué à Albert Camus : « Je préfère ma mère à la justice ».

C'est une première raison pour l'EMC de partir de mythes, contes ou fables, pour développer le jugement normatif et évaluatif si l'on en croit Jerome Bruner ${ }^{9}$ pour qui le récit permet de réfléchir sur le mode "subjonctif ", c'est-à-dire de réfléchir à ce qui pourrait être, à ce qui serait souhaitable, voire à ce qui devrait être.

Nous pensons aussi que le récit permet de jeter un pont « entre ce qui est établi et ce qui est possible ${ }^{10} »$, entre l'indicatif et l'impératif ou le subjonctif, dont les indicateurs traditionnels sont: il était une fois, ce qu'il en est aujourd'hui, ce qu'il pourrait ou devrait en être demain. Le récit est à ce titre le moyen, pour nous enseignants, d'introduire le jeune à la temporalité qui n'est pas celle du temps physique mais celle de l'idéal normatif ou, si l'on préfère, de le faire réfléchir aux questions normatives du savoir-vivre (à ce qu'il convient de faire pour bien faire et pour être juste).

\section{Validité des énoncés}

18 Nous disions plus haut que la validité des énoncés normatifs et prescriptifs, qui constituent le corpus des disciplines de la morale/éthique et du droit, doit être interrogée. En effet, ce n'est pas parce que l'on dit : « Il faut donner plus à ceux qui ont moins » que la phrase est juste et valide pour tous. Cet énoncé se présente comme une prétention à la justesse mais celle-ci doit être éprouvée et recueillir l'assentiment de tous les concernés à l'issue d'une discussion pratique. Telle est du moins ce que Jürgen Habermas préconise dans une visée post-métaphysique dans laquelle le bien et le juste ne se conçoivent pas a priori: «Chaque norme valide devrait pouvoir trouver l'assentiment de tous les concernés, pour peu que ceux-ci participent à une discussion pratique $^{11}$.»

19 Notons que lorsque la validité de ces énoncés n'est pas problématique, ces énoncés peuvent se transmettre assez aisément, même oralement, comme dans les mythes, les contes, les fables et même les proverbes. Ainsi, par exemple, la fable d'Ésope du berger qui criait «Au loup ». Le savoir pratique qu'elle transmet sous la forme d'une «morale de l'histoire ", c'est qu'à mentir quelques fois, nous risquons de ne plus être crus même quand nous dirons la vérité. C'est ce qui permet de dire que la norme « Il faut dire la vérité pour garder la confiance » est valide et peut faire l'objet d'un savoir pratique à transmettre.

20 C'est pourquoi - et c'est une autre bonne raison de s'appuyer sur les mythes, fables, contes et proverbes pour développer le jugement normatif des élèves -, ils constituent des supports pédagogiquement intéressants pour déclencher des réflexions et des discussions éthiques.

21 Cependant à la différence d'hier, nous ne pouvons nous contenter de transmettre un savoir pratique comme une entreprise de moralisation. La modernité politique et pédagogique vise l'éducation au sens de Kant, c'est-à-dire « é-lever » le jeune ou encore le « conduire (ducere) hors de (ex-) » sa minorité, pour en faire un être autonome sur le plan personnel et majeur sur le plan politique. Il s'agit donc, c'est la définition kantienne de l'autonomie, qu'il obéisse volontairement à la règle d'action parce qu'elle se justifie ; et par extension, qu'il lui désobéisse lorsqu'elle ne se justifie pas, ce qui est 
le propre de la désobéissance civile. Et, pour ce faire, le rôle de l'enseignant est d'amener l'élève à retrouver les raisons de la règle qui justifient qu'on lui obéisse. Le savoir pratique doit ainsi être transmis comme un savoir problématique susceptible d'être justifié.

Par exemple, dans le conte «La loi du plus fort? » repris en annexe 2, Tsam-Kui doit-il dire la vérité aux grands bantous qui menacent de s'approprier par la force le bien des pygmées $^{12}$ ? Le conte est l'occasion d'amorcer une discussion sur le droit ou non de mentir. L'enseignant peut alimenter la réflexion en faisant valoir le principe de l'interdiction de mentir, non seulement pour les raisons vues ci-dessus dans la fable d'Ésope, mais plus fondamentalement au sens de Kant: aucune communication humaine ne serait possible si nous ne partions pas du principe de nous dire la vérité ${ }^{13}$ ! En revanche, dans certains contextes et non par principe, des arguments, comme ceux de Benjamin Constant ${ }^{14}$, peuvent être avancés en faveur du mensonge lorsque l'interlocuteur a perdu le droit à la vérité. Que l'on pense aux nazis qui viennent chercher un enfant ou un adulte juifs cachés.

Pour conclure ce point, nous pouvons dire que ce n'est pas parce que l'EMC transmet un savoir pratique que les énoncés normatifs doivent être transmis comme des vérités $a$ priori (moralisme). Il nous faut plutôt amener les élèves à :

- discuter et réfléchir ces énoncés pour qu'ils retrouvent les raisons de la validité d'un énoncé, d'une règle d'action, voire d'un interdit. Rappelons au passage l'étymologie du mot « interdit » : ce qui s'est dit (les raisons) entre (inter) les personnes concernées;

- exercer et développer le jugement normatif (le jugement moral et citoyen ou le jugement qui porte sur les choses qui pourraient être autrement qu'elles ne sont; qui porte sur le devoir-être).

\section{Développer le jugement normatif}

\section{Deux théories du développement du jugement normatif}

Deux grands développementalistes nous ont légué une théorie du développement du jugement normatif, moral et citoyen ${ }^{15}$.

- Jean Piaget dans Le jugement moral chez l'enfant (1932), a mis en exergue trois grands stades du développement : l'anomie, l'hétéronomie et l'autonomie ${ }^{16}$.

$\mathrm{Au}$ premier stade, les tout petits enfants n'ont pas encore intégré les règles du jeu et n'ont pas conscience que la règle est le médium qui permet de jouer ensemble, si bien qu'au jeu, ils ne jouent pas avec les autres, même s'ils jouent à côté des autres enfants :

Jusque vers sept ans, les enfants ne savent guère discuter entre eux et se bornent à

heurter leurs affirmations contraires [...] sorte de "monologue collectif" consistant

à s'exciter mutuellement à l'action plus qu'à échanger des pensées réelles ${ }^{17}$.

Ce stade de l'anomie (en grec : $a$ privatif ; nomos = loi) fait progressivement place, grâce à l'interaction, à la conscience de la règle comme moyen de coordonner l'action ou le jeu avec les autres : «[...] les garçons après sept ans se soumettent de façon beaucoup plus précise et coordonnée à un ensemble de règles communes ${ }^{18}$."

À ce deuxième stade, la règle est donnée par d'autres (hétéros = autre; nomos) mais elle est encore sacralisée, intouchable, incritiquable. Cette hétéronomie du jugement moral n'évoluera en jugement autonome que lorsque l'enfant ne se contentera plus d'obéir à la règle du jeu en tant que telle, parce qu'elle est la règle, mais d'y obéir ou non 
volontairement (auto=soi-même; nomos) ou de la modifier tout aussi librement avec l'accord des autres joueurs.

- Lawrence Kohlberg dans Essays on Moral Development (1981), met au jour trois niveaux $\mathrm{du}$ développement du jugement: pré-conventionnel, conventionnel et postconventionnel, qui recoupent les trois niveaux de Piaget, mais il les décompose en six stades aussi bien pour l'enfant que pour l'adulte ${ }^{19}$.

Ces théories ont été critiquées, notamment parce que leurs adversaires ont confondu développement du jugement moral et moralitér ${ }^{20}$. Nous devons donc dire ici qu'il ne s'agit pas de mesurer la moralité de quelqu'un par le degré de maturité de son jugement. Mais il est utile de posséder un étalon de la croissance morale, celui du jugement. D'ailleurs, l'enseignant n'a pas d'autre choix en classe que de s'adresser au jugement de l'élève, sans jamais pouvoir agir sur son comportement. L'enseignant doit cependant savoir que les études font état d'une bonne corrélation entre le jugement et le comportement et donc qu'en travaillant au développement du jugement normatif, on travaille indirectement à un comportement plus responsable ${ }^{21}$.

\section{Peut-on développer le jugement normatif ?}

30 La réponse est affirmative. Sans être en mesure ici de détailler toute la méthodologie de la recherche, j'ai pu montrer que le jugement moral et citoyen des élèves, qui pratiquent une discussion mensuelle pendant huit mois sur des questions normatives, croît chez des élèves de 5 à 13 ans. La méthodologie de la recherche est exposée dans les numéros $166^{22}$ et $186^{23}$ de la Revue française de pédagogie.

31 La première recherche-action s'est déroulée avec un échantillon de 400 enfants, de 5 à 13 ans, de Bruxelles et du Grand Duché de Luxembourg et de 19 instituteurs (qui avaient été mes étudiants à la Haute École de Bruxelles) répartis en un groupe expérimental et un groupe témoin. Le groupe expérimental a pratiqué pendant huit mois une discussion mensuelle sur des questions normatives tandis que le groupe témoin non. La recherche a permis d'établir une croissance du jugement normatif de $2,9 \%$ au stade $3^{24}(2+1)$ et $2,17 \%$ au stade $4(3+1)$ pour les $7-8$ ans et de $5,09 \%$ au stade $3(2+1)$ et $7,92 \%$ au stade $4(3+1)$ pour les $10-11$ ans.

32 La deuxième recherche-action s'est déroulée avec un échantillon de 50 enfants ${ }^{25}$ de 5 ans d'une école bruxelloise, répartis en deux groupes expérimentaux (34 enfants) et un groupe témoin (16 enfants). Les groupes expérimentaux ont vécu neuf ateliers hebdomadaires de réflexion sur l'identification et la régulation des quatre émotions de base (joie, tristesse, colère, peur), animés respectivement par Chloé Rocourt et moimême, à raison d'une séance de 45 minutes maximum par semaine, tandis que le groupe témoin non. La recherche a permis d'établir une croissance du jugement normatif des enfants des deux groupes expérimentaux. Celle-ci progresse en moyenne de $22,53 \%$ tandis que le jugement normatif des enfants du groupe témoin progresse en moyenne, sur la même période, de $18,75 \%$. Soit un bénéfice de l'expérimentation de $3,78 \%$ en deux mois et demi.

Les deux recherches ne permettent néanmoins pas de dire si cette croissance est durable.

Une autre étude a en tout cas pu montrer que le développement du jugement moral et citoyen connaissait une croissance accélérée entre 11 et $16 \mathrm{ans}^{26}$. 


\section{Littérature et développement}

La fonction narrative serait à la racine même de l'émergence du langage, si l'on suit Bernard Victorri, pour interdire des comportements nuisibles à la survie de l'espèce :

Ainsi, les premiers usages de la fonction narrative auraient consisté à évoquer des crises passées pour interdire des comportements nuisibles à la survie de l'espèce, créant ainsi une organisation sociale totalement inédite dans le règne animal, conduisant à ce que Donald (1991) appelle le stade de la «culture mythique », et c'est ce qui aurait permis à notre espèce d'échapper aux dérégulations sociales ayant conduit à l'extinction des autres Homo sapiens archaïques ${ }^{27}$.

Le langage nous aurait ainsi servi à réguler nos comportements, ce qui est le propre du savoir-vivre, de l'éthique.

\section{La narrativité, une propédeutique à l'éthique}

C'est pourquoi nous pourrions, avec Paul Ricœur, appréhender le récit comme une propédeutique à l'éthique :

[...] il n'est pas de récit éthiquement neutre. La littérature est un vaste laboratoire où sont essayés des estimations, des évaluations, des jugements d'approbation et de condamnation par quoi la narrativité sert de propédeutique à l'éthique ${ }^{28}$.

Tout se passe donc en effet comme si, à la lecture d'une histoire, on était amené à soupeser des valeurs ou à comparer des normes d'actions entre elles et à en privilégier certaines plutôt que d'autres, ce qui est bien le propre des jugements évaluatif et normatif typiques de la moralité.

\section{Performance interprétative : de la conclusion à la cause}

Lorsque le récit se charge des leçons de l'expérience, il peut devenir édifiant. Ainsi en est-il des contes et des fables. Ces textes véhiculent implicitement ou explicitement la «morale de l'histoire ", c'est-à-dire ce qui se passerait si l'on adoptait telle ou telle règle d'action. Le discours se ferait interprétatif selon Jean-Marc Ferry au sens où le récit « au-delà d'une factualité des événements rapportés » mettrait en exergue " la loi qui régit ces événements »; deviendrait « exemplaire ou emblématique, c'est-à-dire représentatif d'un intérêt qui dépasse la singularité historique d'aventures vécues par un héros » en se plaçant « au-delà de la contingence d'événements singuliers ${ }^{29}$ ».

C'est en ce sens que certains pans de la littérature, comme les mythes, les contes, les fables et les récits qui s'en inspirent, peuvent être un point de départ afin que l'enseignant amorce une réflexion et une discussion avec des élèves sur le savoir vivre dans ce qu'il a d'universel.

Philippe Meirieu ne dit pas autre chose :

Les récits sont de véritables cadeaux, les plus beaux cadeaux qu'on puisse offrir aux enfants : ils leur parlent d'eux sans les violer dans leur intimité. Ils les relient, à travers les âges et au-delà des histoires singulières de chacune et de chacun, à la communauté des humains dans ce qu'elle a de plus universel ${ }^{30}$.

L'inflexion de la narration vers l'interprétation suppose, dit encore Jean-Marc Ferry « une participation active du destinataire » et la performance particulière 
qui « consiste ici à transformer la conclusion en cause, en expliquant les événements du récit par un manquement à la loi suggérée par le dénouement ${ }^{31}$ ».

De même, l'enseignant d'aujourd'hui, qui ne peut pas se contenter de transmettre tel quel le contenu pratique de ces histoires, doit pouvoir s'appuyer sur elles pour faire revivre au jeune cette compétence cognitive particulière qui consiste à transformer la morale de l'histoire, la conclusion, en cause, c'est-à-dire en raison d'agir ou non de telle ou telle manière, qui consiste, autrement dit, à inférer, à partir d'un comportement narré, la conséquence funeste qui s'ensuivrait. C'est ainsi une manière d'amener l'élève à comprendre la validité d'une norme d'action pour reproduire ou éviter un événement et d'obéir ou de désobéir librement à une telle norme parce qu'il la juge justifiée.

\section{Le temps de l'idéal}

En outre, comme nous l'avons vu plus haut, les mythes, les contes, les fables et les récits qui s'en inspirent, permettent de jeter un pont entre la réalité et l'idéal, ce qui permet au jeune de prendre conscience d'un idéal régulateur pour l'action morale ou politique. Ces récits sont le moyen pour nous enseignants d'introduire le jeune à la temporalité qui n'est pas celle du temps physique mais celle de l'idéal normatif ou, si l'on préfère, de le faire réfléchir aux questions normatives du savoir-vivre. Ce faisant, ces récits sont aussi l'occasion d'acquérir une autre performance, celle de différencier les modes grammaticaux pour saisir la spécificité du jugement normatif : l'indicatif pour ce qui est, l'impératif pour ce qui doit être et le subjonctif pour ce qui est souhaitable.

\section{Distance propre à la réflexion}

D'un point de vue psychopédagogique, Philippe Meirieu pense que le conte, par exemple, a ceci d'intéressant qu'il permet à l'élève de s'approcher d'une problématique sans se sentir directement et individuellement concerné. Cette distance lui permettrait de réfléchir à la situation sans que des mécanismes de défense se mettent en place pour nier une situation. Ainsi justifie-t-il la lecture du Petit Poucet aux enfants, lecture qui leur permettrait d'apprivoiser des terreurs profondes comme l'abandon des enfants par leurs parents, la peur d'être mangé par l'ogre, « cet être qui, à force de vous aimer et de vous serrer fort dans ses bras vous étouffe et vous dévore irrémédiablement ${ }^{32}$ ».

Le conte permet aussi de respecter le rythme de l'élève :

Car les récits ont cet immense avantage de pouvoir être appréhendés à différents niveaux de profondeur et de complexité : en totale extériorité, en légère tangence ou en s'identifiant plus ou moins fortement avec les personnages ${ }^{33}$.

Le conte permet en outre à l'élève de ne pas résister à ce qui pourrait apparaître comme une moralisation et lui laisse ainsi les coudées franches pour une véritable réflexion:

[...] dans les récits, ce n'est pas un proche qui parle et qu'on pourrait soupçonner de vouloir nous faire la leçon de manière trop directement intéressée, on les écoute avec cette attention un peu flottante qui permet de résonner à ce qu'ils disent sans se sentir, pour autant, agressé $e^{34}$. 


\section{Authenticité expressive}

me semble en tout cas nécessaire pour l'enseignant d'éviter le roman de circonstances et de choisir le récit en fonction de sa qualité littéraire. C'est ainsi qu'il obtiendra que l'élève soit réellement interpellé par le texte. Si l'authenticité expressive n'est pas au rendez-vous, le questionnement, la discussion et la réflexion qui doivent en émerger, risquent d'être de moins bonne qualité, c'est-à-dire plus superficiels, ce qui nous ferait manquer notre objectif. Comme l'écrit Jean-Marc Ferry se référant à Jauss et à Habermas :

Pour être puissant, un récit doit communiquer une grande profondeur d'expérience vécue. Pour cela l'authenticité des sentiments exprimés importe évidemment davantage que l'exactitude des faits rapportés. Outre la précision descriptive, requise pour l'évocation de scènes fortes, l'authenticité expressive apparaît comme la première catégorie de la validité des discours, car c'est elle qui, bien avant la vérité factuelle et la justesse normative, offre les garanties d'une bonne intercompréhension ${ }^{35}$.

En se référant à des histoires, à des textes qui tirent les leçons, convaincantes ou non, d'une expérience vécue supposée, comme le font les mythes, les contes et les fables, l'enseignant bénéficie d'un support pédagogiquement efficace pour exercer le jugement évaluatif et développer le jugement normatif. Par un travail d'interprétation des conséquences d'une règle d'action, l'élève peut en dégager une raison (une cause) de la reproduire ou non. Et cela, non pas en étant moralisé par l'enseignant, mais en participant activement et de façon autonome au processus.

L'intérêt du récit est aussi psychologique dans la mesure où il permet au lecteur de s'identifier au héros de l'histoire sans qu'il se sente directement concerné.

La qualité littéraire du récit sera recherchée pour que l'on soit assuré qu'il interpelle le jeune par des expériences fondamentales et authentiquement expressives.

\section{En guise de conclusion}

Si, d'une part, la spécificité de l'EMC, par rapport aux autres disciplines, vise la transmission d'un savoir pratique - qui doit être validé par l'expérience et/ou la discussion - formé de jugements normatifs (portant sur les normes d'action) et évaluatifs (portant sur les valeurs, les goûts et les préférences) et si, d'autre part, la littérature permet de travailler le devoir-être et le souhaitable, le pont entre l'idéal et la réalité, nous pouvons dire à plus forte raison que les récits des contes et des fables constituent un tremplin à l'éducation morale et citoyenne parce qu'ils véhiculent les leçons de l'expérience sous la forme explicite ou non d'une « morale de l'histoire ».

\section{ANNEXES}

Exemples d'activités pour éduquer le jugement évaluatif et normatif 


\section{Annexe 1. Clarifier le jugement évaluatif}

Clarifier le jugement évaluatif est un objectif qui peut être poursuivi par exemple à partir de l'album de Judy Allen, Dans la forêt vierge ${ }^{36}$.

La finalité de cette activité est de dégager l'un des principes d'une citoyenneté démocratique, à savoir le respect de la pluralité des valeurs (concomitant à la laïcité de l'État).

Après avoir lu l'histoire, et avoir, comme le préconise M. Lipman, recueilli et regroupé les questions des élèves ${ }^{37}$, l'enseignant demande aux élèves de $2^{\text {ème }}$ année (7-8 ans) de repérer tout ce qui est " précieux » pour Karik, le héros de l'histoire. Puisque l'élève n'a pas accès au concept de valeur, l'enseignant va l'aider à le lui faire construire par l'intermédiaire du prédicat " précieux ».

Enfin, les élèves sont invités à créer leur propre échelle de valeurs (à partir des objets précieux), puis à la comparer à celle de Karik, à celle de leurs camarades et à répondre aux questions suivantes :

-L'échelle de nos valeurs est-elle toujours la même?

-Y a-t-il une échelle de valeurs qui est meilleure que les autres?

- Qu'est-ce que Karik aurait mis tout en haut de l'échelle ? Pour quelle raison?

- Peut-on savoir ce qu'est la chose la plus précieuse ? Pourquoi ?

- Si X ou Y avait rapporté son objet le plus précieux au chaman, aurait-il reçu les couleurs sur son front? Pourquoi?

- Quel est le trésor que Karik a ramené dans sa tête?

Une conclusion peut en être dégagée et consignée au cahier comme résultat de l'activité :

Chacun a sa propre échelle de valeurs ; l'une n'est pas meilleure que l'autre.

\section{Annexe 2. Développer le jugement normatif à partir d'un conte.}

La finalité de l'activité consiste à faire réfléchir aux raisons de mentir ou de ne pas mentir.

L'enseignant lit le conte $\mathrm{n}^{\circ}$ 7, « La loi du plus fort ? » de Jan Lantier ${ }^{38}$.

Avec beaucoup de difficultés, Tsam-Kui, le plus vieux des Pygmées, avait convaincu les jeunes de sa tribu de se lancer dans l'agriculture, de défricher un terrain et d'y planter des bananiers. Les jeunes avaient suivi son conseil mais, quand un an plus tard, les bananes arrivèrent à maturité, des Bantous, venus d'un village du Nord, se ruèrent sur la plantation, cueillirent les fruits et empêchèrent les Petits Noirs d'intervenir.

Lorsque l'année suivante, la deuxième récolte s'annonça, les jeunes Pygmées se rendirent chez Tsam-Kui pour lui faire comprendre qu'ils n'allaient pas défendre leur plantation contre les Grands Noirs invincibles :

- Nous ne sommes pas prêts à mourir pour des bananes, lui dirent-ils.

- Laissez-moi faire, leur répondit Tsam-Kui.

Le vieux Pygmée partit à la plantation et y vit, venus d'un village du Sud, des Bantous arrachant les bananes. Tsam-Kui se jeta par terre et commença à pleurer. Il hurla que tous les jeunes de sa tribu étaient morts. Sans y prêter attention, les Grands Noirs continuèrent à cueillir les fruits. Leur chef demanda tout de même au Pygmée ce qui était arrivé aux jeunes de sa tribu. Sanglotant, Tsam-Kui lui répondit: 
- Cette plantation est maudite. Chaque fois que les bananes sont vraiment mûres, le grand troupeau des éléphants géants se précipite dessus et piétine aussi bien les fruits que les hommes qui les récoltent ! Chaque fois, c'est un désastre! Chaque fois, des centaines d'hommes trouvent la mort ici. Allez-vous-en, sinon vous allez périr !

En entendant cela, le chef des Bantous siffla et fit signe à ses hommes de s'encourir. En moins de deux, il n'y avait plus aucun Grand Noir sur la plantation.

En rentrant chez les siens, Tsam-Kui cria :

- Allez récolter les bananes ! Il n'y aura plus jamais de Bantous pour nous les prendre!

L'enseignant, après avoir recueilli deux ou trois questions, anime la discussion. La problématique normative choisie est celle de savoir s'il faut toujours dire la vérité et pourquoi.

À titre d'exemple, voici les questions que j'ai obtenues lors de l'animation d'une $5^{\mathrm{e}} \mathrm{P}$ (10-11 ans) :

$1^{\circ}$. Pourquoi Tsam-Kui dit-il à sa tribu de cueillir les bananes alors qu'il y a encore un danger avec les Bantous du Nord?

$2^{\circ}$. Pourquoi dire « la loi du plus fort »?

$3^{\circ}$. Pourquoi le Tsam-Kui ment-il aux Bantous?

L'enseignant fait répondre aux questions $2^{\circ}$ et $1^{\circ}$ puis cible l'animation autour de la question $3^{\circ}$. Il amorce et élargit la discussion selon le plan de discussion suivant :

- Est-ce bien de mentir ? Oui, non, pourquoi ?

- Imaginez que je dise que demain, il y a un contrôle d'histoire mais que cela n'est pas vrai?

-Quelles seraient les conséquences du fait qu'on ne peut plus faire confiance à personne?

- Si chaque fois que quelqu'un nous adressait la parole, nous pensions qu'il ne dit pas la vérité, que se passerait-il ?

- Connaissez-vous l'histoire du jeune berger qui criait au loup? Que s'est-il finalement passé ?

Conclusion intermédiaire : le mensonge nuit à l'humanité (à la communication, à la confiance, au contrat... et donc, comme le dit Kant, à la source du droit).

- Dans le conte que nous venons de lire, pensez-vous que Tsam-Kui a raison de mentir? Oui, non, pourquoi?

Lire la citation de Benjamin Constant, réagissant à Kant, qui dit, en 1797 (dans Réactions politiques, p. 36) :

Dire la vérité est un devoir. Qu'est-ce qu'un devoir ? L'idée de devoir est inséparable de celle de droits : un devoir est ce qui, dans un être, correspond aux droits d'un autre. Là où il n'y a pas de droits, il n'y a pas de devoirs. Dire la vérité n'est donc un devoir qu'envers ceux qui ont droit à la vérité. Or nul homme n'a droit à la vérité qui nuit à autrui.

- Pensez-vous que ce que Constant nous dit s'applique au conte? Que Tsam-Kui ne doit pas la vérité aux Bantous? Oui, non, pourquoi?

-Vous est-il déjà arrivé, de devoir mentir pour préserver votre droit? 
L'enseignant amène les élèves à généraliser le propos en rédigeant une sagesse du jour (un savoir pratique) qui synthétise la discussion. De façon à ce que l'élève formule un jugement normatif, il lui est demandé d'écrire sa sagesse sous la forme d'un conseil.

À titre d'exemple, voici les sagesses que j'ai recueillies dans la classe de $5^{\mathrm{e}} \mathrm{P}$ (10-11 ans) dont nous avons lu les questions plus haut :

- Ne pas mentir sauf exception! (3 voix)

- Ne pas mentir sauf quand une vie est en danger (9 voix) (sagesse votée par la classe)

- Mieux vaut dire la vérité sauf en cas de danger (3 voix)

- Dans certains cas, il ne faut pas mentir (0 voix)

- Ne mens pas pour éviter de te sentir mal (1 voix)

- Mieux vaut dire la vérité ( 0 voix)

L'activité de transfert ou d'intégration cognitive consiste à faire réfléchir les enfants tout au long de l'année scolaire sur ce point : en cas de mensonge, vérifier s'il se justifie par une atteinte au droit.

\section{NOTES}

1. Qui veut dire règle.

2. On retrouvera ici à gros traits les trois impératifs kantiens (E. Kant, Critique de la raison pratique [1788], trad. F. Picavet, Paris, PUF, coll. «Quadrige», 1943, p. 18). Voir les trois types de discussion pour valider ces impératifs chez Jürgen Habermas: De l'éthique de la discussion, trad. M. Hunyadi, Paris, éd. du Cerf, 1992, p. 102.

3. Pour un plus long développement, voir Claudine Leleux, Hiérarchiser les valeurs et les normes de 5 à 14 ans, Bruxelles, éd. De Boeck et Van In, coll. « Apprentis citoyens », 2014, p. 60-66.

4. Voir la leçon $n^{\circ}$ 5, « Préférer », ibid., p. 74-78.

5. Voir annexe 1. «Clarifier le jugement évaluatif».

6. Voir la leçon $n^{\circ} 9$ dans Claudine Leleux, Chloé Rocourt, Pour une didactique de l'éthique et de la citoyenneté. Développer le sens moral et l'esprit critique chez l'adolescent, Bruxelles, éd. De Boeck et Van In, 2010, p. 179-198.

7. Par exemple et à remettre dans son contexte: «Je ne veux pas que l'on me mêle à ces prêcheurs de l'égalité et que l'on me confonde avec eux. Car c'est ainsi que la justice me parle à moi : "Les hommes ne sont pas égaux" » (Friedrich Nietzsche, Ainsi parlait Zarathoustra, trad. G.A. Goldschmidt, Paris, Livre de poche, $\mathrm{n}^{\circ}$ 987, 1972 [1883], p. 138).

8. Pour un développement de la question, voir Cl. Leleux, Chl. Rocourt, J. Lantier, Éducation à la philosophie et à la citoyenneté. Didactique et séquences, Bruxelles, De Boeck \& Van In, 2017, p. 43-46.

9. J. Bruner, Pourquoi nous racontons-nous des histoires? Le récit au fondement de la culture et de l'identité, Retz, 2010 [2002], p. 89-90.

10. Ibid., p. 16.

11. J. Habermas, De l'éthique de la discussion, trad. M. Hunyadi, Paris, éd. du Cerf, 1992 [1991], p. 34. 12. Conte $\mathrm{n}^{\circ} 7$ de Jan Lantier dans Cl. Leleux, J. Lantier, Discussions à visée philosophique à partir de contes de 5 à 14 ans, Bruxelles, éd. De Boeck et Van In, coll. «Apprentis philosophes », 2010, p. 69-75.

13. Voir Emmanuel Kant, Sur un prétendu droit de mentir par humanité, trad. L. Guillermit, Paris, Vrin, 1990 [1797], p. 69 : « [le mensonge] nuit toujours à autrui : même si ce n'est pas à un autre homme, c'est à l'humanité en général, puisqu'il disqualifie la source du droit. » 
14. Voir Benjamin Constant, «Réactions politiques", dans De la force du gouvernement actuel de la France et de la nécessité de s'y rallier, Paris, Flammarion, 1988 [1797], p. 36 : «Dire la vérité est un devoir. Qu'est-ce qu'un devoir? L'idée de devoir est inséparable de celle de droits : un devoir est ce qui, dans un être, correspond aux droits d'un autre. Là où il n'y a pas de droits, il n'y a pas de devoirs. Dire la vérité n'est donc un devoir qu'envers ceux qui ont droit à la vérité. Or nul homme n'a droit à la vérité qui nuit à autrui. » [En ligne] : <http://classiques.uqac.ca/classiques/ constant_benjamin/des_reactions_politiques/des_reactions.html>

15. Pour un plus long développement, voir $\mathrm{Cl}$. Leleux, Ch. Rocourt, Pour une didactique, ouvr. cité, p. 65-78.

16. J. Piaget, Le Jugement moral chez l'enfant, Paris, PUF, coll. «Bibliothèque philosophie contemporaine », 1995 [1932].

17. J. Piaget, "Le développement mental de l'enfant» dans Six études de psychologie, Paris, coll. « Folio essais », 1964, [1940], p. 34.

18. Ibid. p. 81.

19. L. Kohlberg, Essays on Moral Development, vol. 1, Philosophy on Moral Development, San Francisco, Harper \& Row, 1981.

20. J'ai notamment critiqué chez Kohlberg la séparation entre les stades 5 et 6 . Voir Cl. Leleux, "Théorie du développement moral chez Lawrence Kohlberg et ses critiques (Gilligan et Habermas) ", dans J.-M. Ferry et B. Libois (dir.), Pour une éducation postnationale, Bruxelles, éd. de l'Université de Bruxelles, 2003, p. 111-128. [En ligne] : <http://users.skynet.be/claudine.leleux/ KohlbergPublDEA.pdf>

21. Voir par exemple M. Rainville, Manuel pratique de formation à l'approche de Kohlberg, Université du Québec, 1978, p. 62.

22. Cl. Leleux, «La discussion à visée philosophique pour développer le jugement moral et citoyen? ", La Revue française de pédagogie, $\mathrm{n}^{\circ}$ 166, Paris, janvier-mars 2009, Institut National de la Recherche Pédagogique, p. 71-87. [En ligne] : <https://journals.openedition.org/rfp/1271>

23. Cl. Leleux, «Discussions à visée philosophique pour développer le jugement normatif des 5 à 13 ans. Recherche-action, problèmes méthodologiques et résultats", La Revue française de pédagogie. Recherches en éducation, $\mathrm{n}^{\circ} 186$, Paris, janvier-mars 2014, Institut français de l'Éducation, p. 75-84. [En ligne] : <https://journals.openedition.org/rfp/4410>

24. Fait référence aux stades de l'échelle du développement du jugement moral chez L. Kohlberg. 25. Seulement ceux qui étaient présents à la fois au pré-test et au post-test, soit entre le 29/09/11 et le $15 / 12 / 2011$.

26. J. Lalanne, «Le développement moral cognitif chez Lawrence Kohlberg » dans Entre-vues, $\mathrm{n}^{\circ} 7$, 1990, p. 18 : «L'adolescence (de 11 à 16 ans) est une période de développement moral accéléré. On observe en particulier une différence importante entre les jeunes de 11-12 ans et les adolescents de 14-16 ans. La moralité autonome se développe entre 12 et 16 ans. Aucune différence significative ne peut être retenue entre garçons et filles de 10 à 17 ans. Le passage du niveau préconventionnel au stade conventionnel s'opère généralement en Amérique juste avant l'adolescence et au niveau post-conventionnel vers la fin de l'adolescence, soit de 15 à 19 ans ».

27. B. Victorri, «Homo narrans : le rôle de la narration dans l'émergence du langage ", Langages, $\mathrm{n}^{\circ}$ 146, 2002, p. 122. [En ligne] : <http://www.persee.fr/web/revues/home/prescript/article/ lgge_0458-726X_2002_num_36_146_2406>, consulté le 15 mai 2016, p.116. Victorri renvoie au livre de Merlin Donald Origins of Modern Mind: Three Stages in the Evolution of culture and Cognition, Harvard University Press, 1991 (trad. française: Les origines de l'esprit moderne, De Boeck Université, 1999).

28. P. Ricœur, Soi-même comme un autre, Paris, Seuil, 1990, p. 139.

29. J.-M. Ferry, Les puissances de l'expérience, vol. 1, Le sujet et le verbe, Paris, éd. du Cerf, 1991, p. 112. 
30. Ph. Meirieu, «Même pas peur de la peur!», p. 5. [En ligne] : <http://www.meirieu.com/ ARTICLES/La_peur.pdf>, site consulté le 17 février 2013.

31. J.-M. Ferry, ouvr. cité, p. 115.

32. Art. cité p. 4.

33. Ibid. p. 5.

34. Id.

35. Ouvr. cité, p. 106-107.

36. J. Allen, Dans la forêt vierge, trad. P. Bertrand, ill.J. Butler, Paris, Paris, L'École des loisirs, 1995.Voir la Leçon $n^{\circ} 6$ de Karin Molter dans Cl. Leleux, Hiérarchiser des valeurs et des normes, 2014, ouvr. cité, p. 79-85.

37. Voir par exemple M. Gimenez-Dasi, M.-F. Daniel, Réfléchir sur les émotions à partir des contes d'Audrey-Anne pour les 4 à 8 ans, Bruxelles, De Boeck et Van In, coll. «Apprentis philosophes », 2012, p. 15-18.

38. Extrait de Cl. Leleux, J. Lantier, Discussions à visée philosophique à partir de contes de 5 à 14 ans, Bruxelles, éd. De Boeck et Van In, coll. « Apprentis philosophes », 2010, p. 69-75.

\section{RÉSUMÉS}

La présente contribution vise à montrer que les fables et les contes, parce qu'ils appartiennent à un genre littéraire particulier, sont des supports idéaux à partir desquels l'enseignant peut développer le jugement moral et citoyen des élèves et ainsi rencontrer l'une des finalités de l'Enseignement moral et civique (EMC). Ce sont des récits édifiants qui condensent les leçons de l'expérience et fournissent à l'auditeur puis au lecteur un savoir pratique. Dire cela revient à montrer qu'il y aurait en morale un savoir et même une certaine vérité à transmettre. De quel type de savoir s'agit-il dès lors qu'il est question de valeurs et de normes d'action? De quelle vérité peut-on parler dans ce cas? Et cette vérité serait-elle a priori ou, au contraire, a posteriori? Autant de questions que l'auteur aborde dans cet article sans négliger les questions pédagogiques qui en découlent.

This contribution intends to show that fables and tales, belonging to a specific type of literature, are perfect tools for teachers which help them to develop the moral and the citizen judgment of their pupils and attain in this way one of the objectives of the moral and citizen education. These edifying narratives condense the lessons of experience and give the listener, afterwards the reader, a practical knowledge. Stating this means that these "morals" contain a certain knowledge and even a certain truth to communicate. What kind of knowledge is here at stake since we are talking about norms and values of action? What kind of truth are we facing in this case? Is it an a priori truth or, on the contrary, an a posteriori truth? In this article, the author deals with all these questions without neglecting their pedagogical consequences. 


\section{AUTEUR}

\section{CLAUDINE LELEUX}

Claudine Leleux est philosophe de formation et auteur d'une vingtaine d'ouvrages. Elle s'est spécialisée dans la didactique de la morale non confessionnelle et de l'éducation à la citoyenneté. Elle est actuellement en charge de la formation au Certificat de didactique du cours de philosophie et de citoyenneté à la Haute École Bruxelles-Brabant. Voir <users.skynet.be/ claudine.leleux> 